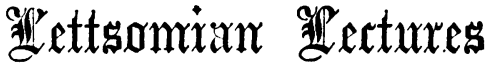

ON

\section{THE SURGICAL DISEASES OF CHILDREN.}

DELIVERED BEFORE THE MEDICAL SOCIETY OF LONDON.

BY

TH O M S B R Y N T, F.R.C.S., ASSISTANT-SURGEON TO GUY'S HOSPITAL.

\section{Lecture III. (Concluded.)}

\section{ON TUMOURS IN CHILDREN.}

The pathological nature of the tumours found in children differs but little from those in the adult. They may possess peculiarities which are due to the rapid growth and structure of all infantile tissues; but, in their morbid and natural pathology, they are strictly analogous. There are the simple or innocent, and the malignant or cancerous tumours. In both forms, fibre-tissue is deficient, and cell-development abundant. Of the simple tumour we have, therefore, the fibro-plastic or fibrocellular more frequently than the fibrous; and of the cancerous, the carcinoma medullare rather than the carcinoma fibrosum.

Tumours also, when they appear in any gland or tissue, as a rule have a rapid growth; the increase of the tumour keeping pace with the increase of the body; the cell-growth and multiplication of the abnormal equalling the cell-growth and development of the normal tissues.

In the bones of children there seems to be a special tendency to the development of tumours ; cartilaginous grow ths appearing in these parts more frequently at an early period of life than at a late one. Cartilaginous or enchondromatous tumours of bone are almost always found in children ; and, when in the adult, they have generally originated in early life. The explanation of this fact does not appear difficult, when we recollect that bony development almost always proceeds through the cartilaginous; for the cartilagecell has but to remain as such, and to go on repeating itself, instead of progressing towards the development of a bone-structure, in order to form a tumour. This cartilage or enchondromatous tumour, under these circumstances, appears developed within a bone, and expands its shell. A most beautiful instance of this disease has recently come under my care; it was in a woman aged 32 , and was of congenital growth, its increase having been gradual. It occurred in the little finger, and was treated by amputation. The specimen I now hand round.

Congenital Tumours. One of the most frequent forms of congenital tumours is the sebaceous, and its most common seat is the neighbourhood of the eyebrow. These sebaceous tumours have also one peculiarity : they are not situated in the skin, as are the other forms, but are placed beneath the muscle and close upon the bone. They very generally contain hair, in addition to the ordinary sebaceous matter; and this is found either in masses, or like fine eye- lashes. These sebaceous tumours may appear also in other parts. I removed one from the labium of a child four months old, which contained abundant hair ; and a second, of the size of a walnut, from the extremity of the coccyx of a girl aged 10 years.

Congenital fatty tumours are not unfrequent; and they occasionally possess a peculiarity for which it is difficult to account. In adults, fatty tumours are, as a rule, encysted; that is, they are distinct tumours, and can be enucleated. In children, an opposite condition is not infrequently found t the fatty tumour appearing as a diffused growth, and without distinct limits. I had an instance of this under my care in 1857, in a boy aged six weeks. He had a diffused congenital fatty tumour around the right elbow-joint, and this grew with his growth. It had no boundary, nor any borders ; but its margins gradually disappeared in the tissues around. I: have witnessed a second case in a child aged 5 , a patient of Mr. Cock's ; and in this instance the region of the occiput was the seat of the disease. I have also under care at the present time a child aged one year and a half, with the same affection; the tumour occupying the left axilla and side of the chest.

Congenital pedunculated fibro-cellular tumours of the integument are very common, and need no description. The best example I have ever seen was in a male child who came under my care a few months ago, when only three days old, with a pedunculated tumour, of the size of a large walnut, growing behind the left ear; it was black from effused blood, and was very firm. It was excised, and recovery followed. The preparation and drawing, which I hand round, indicate its size and nature. An interesting case has also lately been under my care, of congenital fibro-plastic tumour growing from the little toe of a girl aged 8 ; it was of the size of a walnut, and occupied the whole of the extreme phalanx of the toe. It was amputated; and the specimen I now hand round.

Congenital cancerous growths also occur, but it has never fallen to my lot to witness an example.

Non-congenital Tumours: Sebaceous Tumours. One of the most singular cases of sebaceous tumour which has ever come under my care was in a female child aged five months, whose face and head were literally studded with sebaceous tumours, varying from the size of a hempseed to a large almond. They had been of three months growth, and were in all stages of development; some just appearing, others suppurating; and some had enucleated their contents, and presented excavated granulating surfaces. I removed many by pressure with the nail, and the larger by incision; giving tonics, and maintaining cleanliness. Recovery followed.

The fatty and fibro-cellular and fibro-plastic tumours need not occupy our attention, as they present no points of contrast or peculiarity which deserve notice. But I cannot refrain from quoting a case of simple cystic tumour which came under my care in 1861. It was in a boy aged six weeks, and was, when seen, of about the size of a small egg, situated on the right side of the median line over the occiput. It was evidently cystic, and appeared to be connected solely with the soft tissues. It was observed the day after its birth, and was believed to have been congenital; and was then of the size of a small nut. I tapped it, and drew off some fluid stained with blood; and the cyst collapsed. When seen 
three weeks afterwards, no appearance of a re-collection could be observed. I have no later observation to record.

Keloid Tumours. These do not appear to be infrequent growths during young life. I have seen several such. One was in a male aged ten years. The keloid tumour was over the forehead, and was of the size of a nut. It was said to have been congenital, and was growing. It was excised. Recovery followed. I have also observed the same disease in a boy six months old, growing from the cicatrix on a spot from which I had removed a nævus by ligature some months previously. The same disease appeared also in the child of a cousin of the former patient, in the cicatrix of a nævus which I had similarly treated.

Cancerous Growths are not uncommon in young life, and these appear to attack the bones more frequently than other parts. The best example which I have seen was in a boy aged twenty months. The tumour was in the head, and occupied the position of the metacarpal bones, spreading the tendons over it. It was globular, as seen in the drawing and preparation; and was, as usual, of a medullary character. The glands were uninvolved. No other treatment than amputation could be thought of ; and this I did in September 1859. In J862, the child was well, and had had no return.

Tumours of the Testes. Tumours of the testes must occupy our attention, although only for a few minutes. I have the records of one interesting case of inflammation of both organs in a boy six months old, who had had an enlargement of both glands from birth. When I first saw the child, the right was of the size of an unshelled almond, and the body of the testis was the part involved; and the left was about half the size of the right. I regarded the case as inflammatory, although no history of injury or of syphilis could be obtained; and gave tonics. In one month the right testis suppurated, and subsequently recovered; the left gradually became smaller.

I have also had under my care a child only six weeks old, with an abscess in the right testis of a day's duration, after inflammation a week old. No appearances of injury were visible, although, from the rapidity of the attack and progress of the disease, perforation with a pin appeared probable.

Respecting cancerous disease, I can relate an interesting case of a boy agcd two years, who came under my care at Guy's Hospital in February 1860, with medullary cancer of the right testis of six months' growth. The organ had enlarged gradually for four months, and for the last two had increased more rapidly. When excised, it was of about the size of a small bantam's egg. On April 3rd, I removed the gland; and a speedy recovery followed. He remained well till May 1862 , nearly two years, when a retuin growth appeared at the original seat; and this increased rapidly for three months, when I again saw him. At this date, there was a tumour of the size of a walnut in the position of the original testicle : it appeared moveable, and gave but little pain. No glandular enlargement could be detected. On August 6th, 1862, I again excised a portion of the growth, but found that a narrow neck of it dipped downwards into the perinæum, which could not be removed The wound healed kindly, but the growth increased; and in November 1862, when I last saw the child, he was sinking from apparently cancerous infiltration of the lumbar glands.

Strumous disease of the testes may also be found during young life. I have seen it in a child two years old, and of one year's growth; and again in a second aged $2 \frac{1}{2}$, in which the disease had existed six months. In the last case, excision was called for ; and the gland contained a large mass of strumous deposit, which was softening down. Recovery followed. Both of these cases were under my own care.

Inflammation of the Sterno-Mastoid Muscle. I must not omit to dwell upon a class of cases to which attention has not been much drawn, although it can hardly be doubted that most surgeons must hare had such instances under their charge, although their true nature does not appear to have been understood. I allude to thickening and enlargement of the body of the sterno-cleido-mastoid muscle. I have observed it in three separate instances; and have always, from the ill-defined nature of the swelling, looked upon it as inflammatory.

CASE I. A boy, aged 3 months, was brought to me in 1857. The mother stated that the disease had appeared three weeks, and had gradually increased. The muscle was hard and much thickened; and, on manipulation, the child gave evidence of pain. I gave cod-liver oil and ordered warm water fomentation, which rapidly dispersed the disease.

CASE Ir. A female child, aged 2 months, came under my care in 1858. The disease was of one week's standing, and was of the same nature as in the last case. It was cured also by the same treatment.

CASE III. This case occurred in 1862, and was in a male child aged 5 months. The disease had existed five weeks, and was very marked; the body of the muscle stood out like a distinct tumour, but it had no defined boundary. Quinine and fomentation proved rapidly successful, and a cure resulted.

In none of these cases could any history of injury be traced. The disease was in all alike; it was in the sterno-mastoid muscle, and was evidently inflammatory ; the inflammatory product apparently infiltrating the body of the muscle.

My colleague Dr. Wilks has recently reported several cases of a like kind; but, with these exceptions, I have no other record of the affection.

Tumours at the Umbilicus. The fleshy tubercle at the umbilicus is an affection which occasionally comes under notice; it is evidently composed of nothing more than exuberant granulations, as it always follows the separation of the umbilical cord, and is readily removed by the application of a ligature. I have had five cases under my care within the last few years in children, aged respectively fifteen, six, and five months, seven and five weeks; and in all a cure was at once effected by ligaturing the growth.

In connexion with this subject, I cannot refrain from quoting the brief note of an interesting case of growth from the umbilicus, of a totally different nature from those I have described. It was in a boy aged 8 years, who had a fleshy growth springing from the umbilicus, precisely like the glans penis of an infant. It was very red, and covered with mucus; and in its centre there was a distinct canal, through which urine passed; when the child retained its 
urine, it flowed freely from the opening ; for it appeared tolerably certain that the canal was an open urachus. I was anxious to admit the child into Guy's, to see if anything could be done for his relief; but the mother refused, and I consequently had no opportunity of carrying out my wishes.

Cases like these are very rare, and are, therefore, worthy of a separate record.

I have thus, in these three lectures, brought before you the principal points of difference between the diseases of the various systems in the child and adult; and have, as far as I have been able, dwelt upon many of the special affections of early life. I have attempted to find an explanation of these differences, in the physiological and pathological processes as witnessed at these different periods of existence ; and I have aimed at giving principles, rather than details of daily practice. How far I have succeeded I must leave to your kind judgment to decide. But of this I am sure, that I have advanced nothing which experience has not sanctioned; and, if I have been too short and brief in many of my descriptions, I have been so from the necessity which has been always before me of confining my observations to the few hours which are allotted to these Lettsomian Lectures. In conclusion, I have to express my thanks for the kind attention with which you have followed me ; and to assure you that, if I have added anything either of interest or of fact to the important subject which has occupied our notice, I have been amply repaid, and I shall feel that the object for which these lectures were instituted has not suffered from having been intrusted to my care.

\section{OBriginal Commanitations.}

\section{CASE OF RENAL CALCULUS.}

By W. H. DAY, M.D., L.R.C.P.Lond., Newmarket.

In the British Medical Journai, for June 6th, 1863, Dr. John Brown of Rochester has related a very instruc. tive case of renal calculus. After death, "four calculi and several ounces of thin pus" were found in the left lidney, and there was a large cyst in the right kidney. A case of renal calculus, beset at one time with ob. scurity in diagnosis, and attended with great danger to life, has lately come under my observation. I extract the following report from my case-book.

Mr. R., aged 59, a tall, well-formed man, of regular and active habits, and seldom ailing, except from occasional constipation, which usually yielded to a domestic remedy, was attacked on the evening of Oct. 8th, 1862, with severe stabbing pain in the left lumbar region, so that he could not keep in one position for a minute together. He vomited dark green bile, and could retain nothing on his stomach. His suffering was referred to the left side, between the crest of the ilium and the lower ribs. The pain was very acute, extending across the lumbar region to the edge of the spine; the pulse was quick and sharp; countenance most painfully anxious; tongue brown and dry. The abdomen was clear on percussion over the crecum and region of the colon; but some dul. ness was detected at the umbilicus. The urinary secretion was scanty and high coloured, and the bowels had not acted for two days. T'en grains of calomel were placed on the back of the tongue, turpentine stupes were applied to the side, and a turpentine and castor oil enema thrown up the rectum.
On the 9th, he was worse ; sickress had continued un. interruptedly during the night, and there had been no re. lief of the bowels; pulse 120. A cathartic enema was ordered, which returned without any tinge of fæcal matter. Ten leeches were applied to the side; six grains of calomel and one drop of croton oil were given; and small doses of the sulphate and carbonate of magnesia, with syrup of ginger and dill water, were also ordered.

On the 10th, I fourid him much worse. The belly was tympanitic, and tender on pressure; his knees were drawn up, and his breathing was entirely thoracic; vomiting and hiccup were constant; and he could not keep his medicine down.

I was fearful, from these symptoms, that there was either internal strangulation, or very severe spasmodic stricture at or near the splenic curve of the colon, producing colic in its most severe form. I was impressed with the belief that in cases of obstinate constipation, with pain and sickness, purgatives are apt to become eventually irritants; I omitted the medicine prescribed the day before, and gave the citrate of soda in efferves. cence, with small doses of nittous ether and prussic acid.

On the 1lth, the bowels acted gently, and the vomiting and pain were relieved. Late in the evening, it seemed so probable that focal matter was the main exciting cause in operation, that I determined to try two pills every four hours, made with two grains of extract of oxgall, two grains of aqueous extract of aloes, and two grains of extract of hyoscyamus.

On the 12th, the bowels acted most copiously; and, although there was great tenderness in the left renal and splenic region, he was so much relieved, that I took my departure, after advising two of the pills twice a-week, and a dose of castor oil in the morning if they should effectually clear the bowels. He was to take a warm bath every night at bed-time.

He went on well till November 6 th, when I was again asked to see him. A similar train of symptoms pre. vailed, and vomiting was again urgent. I now began to suspeci the presence of a calculus in the left kidney; the bowels being undisturbed, and the pain extending in the course of the ureter.

On the 18th, he was cupped over the left lumbar region, and copious enemata of warm water were thrown up the bowels night and morning.

On the 19th, he was no better. Fearing that the case might lead to disorganisation in the kidney-although no enlargement could be detected, and there was an absence of pus and blood in the urine-I met Mr. Image of Bury St. Edmunds in consultation. He thought there was a calculus in the kidney, and advised a continuance in the treatment, particularly the enemata, to which the patient had a great objection.

A fortnight later, after despairing of recovery, and being very worn and sallow, he passed a calculus of phosphate of lime of the size of a horse-bean. From that hour, he rapidly gained his health and strength.

RFMarks. This case commenced with symptoms of spasmodic constriction in the splenic curve of the colon, or of strangulation at this part. No evidence existed of renal mischief, beyond the sickness and lumbar pain; there being no frequency in micturition, no trace of blood or pus in the urine, and no fever.* Such obstinate constipation is not usual where the intestines are not involved. This, no doubt, partially kept up a state of hyperæmia in the kidney, favourable to pain and vomiting, and unfavourable to the descent of the calculus.

* The pain of colic is often associated with sickness and retching and it may occupy those parts of the abdomen which correspond $t$, and it may occupy those parts of the abdomen which correspond $t$,
the place of the ureters. The urinary functions are undisturbed, an: the place of the ureters. The mrinary functions are undisturbed, and
this is a capital point of distinction. The numbness of the thigh and drawing up of the testicle are sufficiently characteristic when they happen, but they are frequently altogether absent. (Dr. Watson, Principles and Practice of Nedicine, vol. ii, p. 617.) 